June 1996

HU Berlin-EP-96/21

\title{
Gauge fixing, zero-momentum modes and the calculation of masses on a lattice e
}

\author{
V.K. Mitrjushkin ${ }^{1}$ 光 \\ ${ }^{1}$ Institut für Physik, Humboldt-Universität, 10115 Berlin, Germany
}

\begin{abstract}
It is shown that the zero-momentum modes can strongly affect the values of the masses, for example the magnetic screening mass $m_{m}$, calculated from gauge-dependent correlators with zero momentum.
\end{abstract}

The lattice approach gives the possibility to calculate numerically gauge invariant objects without gauge fixing. However in many practical situations it is rather useful to calculate gauge dependent quantities. For example, calculating the (gauge-variant) gluon correlators one can attempt to obtain information about gauge-invariant observables, like energies and masses.

The usual way to proceed is the following [1]. Define zero-momentum operators $\mathcal{O}_{\mu}(\tau)$ :

$$
\mathcal{O}_{\mu}(\tau)=\sum_{\vec{x}} \mathcal{O}_{\mu}(x) ; \quad \mathcal{O}_{\mu}(x)=\frac{1}{2 i}\left(U_{x \mu}-U_{x \mu}^{\dagger}\right),
$$

where $U_{x \mu} \in S U(N)$ are link fields. The symbol $\tau$ means the separation in one of the four euclidian directions, and $\vec{x}$ corresponds to the three complementary directions. Choose the Lorentz (or landau) gauge by maximizing the trace of the link fields $\mathcal{G} \equiv \sum_{x \mu} \operatorname{Tr}\left[U_{x \mu}+U_{x \mu}^{\dagger}\right]$, and calculate the (connected) correlator

*Work supported by the Deutsche Forschungsgemeinschaft under research grant $\mathrm{Mu}$ $932 / 1-4$

${ }^{\dagger}$ Permanent adress: Joint Institute for Nuclear Research, Dubna, Russia 


$$
\Gamma_{\mu}(\tau)=\left\langle\operatorname{Tr}\left(\mathcal{O}_{\mu}(\tau) \mathcal{O}_{\mu}(0)\right)\right\rangle
$$

The effective masses $\tilde{m}(\tau)$ are defined as

$$
\frac{\cosh \tilde{m}(\tau)\left(\tau+1-\frac{1}{2} N_{4}\right)}{\cosh \tilde{m}(\tau)\left(\tau-\frac{1}{2} N_{4}\right)}=\frac{\Gamma_{\mu}(\tau+1)}{\Gamma_{\mu}(\tau)} .
$$

At zero temperature in the confinement phase the large $-\tau$ behaviour of $\tilde{m}(\tau)$ is supposed to describe the gluon mass $m_{g}$ if $\tau$ is chosen along the 'temperature' direction $x_{4}$. At high temperatures in the chromoplasma phase the long distance behaviour of $\Gamma_{1 ; 2}(z)$ and $\Gamma_{4}(z)$ is expected to determine the magnetic and electric screening masses $m_{m}$ and $m_{e}$, respectively (see, for example, [2]).

The translation-invariant gauge fixing, e.g. Lorentz gauge condition, does not exclude the appearence of the zero-momentum modes in the periodic volume. These modes are usually assumed to be non-important. Indeed, their contribution to the Wilson loops is rather small, at least in the weak coupling region [3]. It is the aim of this note to discuss the possible influence of the zero-momentum mode on the gauge-dependent (zero-momentum) correlators.

In what follows $N_{\mu}$ means the size of the lattice in the direction $\mu$, and $V_{4}=N_{1} N_{2} N_{3} N_{4}$. Boundary conditions are periodic, and the lattice spacing is chosen to be unity. The lattice derivatives are: $\partial_{\mu} f(x)=f(x+\hat{\mu})-f(x)$ and $\bar{\partial}_{\mu} f(x)=f(x)-f(x-\hat{\mu})$.

Throughout this paper the restriction to small quantum fluctuations $A_{x \mu}$ about some constant value is implicitly presumed.

It is rather instructive to consider first the $U(1)$ gauge theory. The photon is massless in the Coulomb phase, and the perturbation theory is expected to be reliable in the weak coupling limit.

In this case $U_{x \mu}=\exp \left(i \theta_{x \mu}\right)$ with $\theta_{x \mu} \in(-\pi ; \pi]$, and the Wilson action $S(\theta)$ and the partition function $Z$ are

$$
\begin{aligned}
S(\theta) & =\frac{1}{g^{2}} \sum_{x} \sum_{\mu \nu=1}^{4}\left(1-\cos \theta_{x ; \mu \nu}\right) ; \\
Z & =\int\left[d \theta_{x \mu}\right] e^{-S(\theta)}, \quad\left[d \theta_{x \mu}\right]=\prod_{x \mu} \frac{d \theta_{x \mu}}{2 \pi},
\end{aligned}
$$


where $\theta_{x ; \mu \nu}=\partial_{\mu} \theta_{x \nu}-\partial_{\nu} \theta_{x \mu}$. The action is invariant with respect to the gauge transformations $U_{x \mu} \stackrel{\Omega}{\longrightarrow} U_{x \mu}^{\Omega}=\Omega_{x} U_{x \mu} \Omega_{x+\mu}^{\dagger} ; \quad \Omega_{x} \in U(1)$.

The standard perturbation approach reads as follows (see, for example, (4). Define the Faddeev-Popov determinant $J(\theta)$ :

$$
1=J(\theta) \int \prod_{x} d \Omega_{x} \prod_{x} \delta\left(F_{x}\left(\theta^{\Omega}\right)\right)
$$

where $F_{x}(\theta)$ is some gauge fixing functional. Setting it equal to

$$
F_{x}(\theta)=\sum_{\mu} \bar{\partial}_{\mu} \sin \theta_{x \mu}-C_{x}
$$

inserting the identity (6) in the integral of eq.(5), multiplying both sides by $\prod_{x} \exp \left(-\frac{1}{\alpha g^{2}} C_{x}^{2}\right)$ and integrating over $C_{x}$ one obtains

$$
Z \sim \int\left[d \theta_{x \mu}\right] e^{-S_{e f f}(\theta)} ; \quad S_{e f f}=S+S_{g f}+S_{F P}
$$

where $S_{g f}=\frac{1}{\alpha g^{2}} \sum_{x}\left(\sum_{\mu} \bar{\partial}_{\mu} \sin \theta_{x \mu}\right)^{2}$, and $S_{F P}$ is the contribution from the corresponding Faddeev-Popov determinant to the Lorentz gauge. The average of any (gauge invariant or not) functional $\Phi(\theta)$ is defined as

$$
\langle\Phi\rangle=\frac{1}{Z} \int\left[d \theta_{x \mu}\right] \Phi(\theta) \cdot e^{-S_{e f f}(\theta)}
$$

Making the substitution $\theta_{x \mu}=g A_{x \mu}$ with $A_{x \mu} \in\left(-\frac{\pi}{g}, \frac{\pi}{g}\right]$, expanding in powers of $g^{2}$ and extending the limits of integration to $[-\infty ; \infty]$ one recovers standard perturbation theory on a lattice with a free action $S_{\text {eff }}^{(0)}$ :

$$
S_{e f f}^{(0)}=\sum_{x y} \sum_{\mu \nu} A_{x \mu} L_{x y}^{\mu \nu} A_{y \nu} ; \quad L_{x y}^{\mu \nu}=\left[\left(1-\frac{1}{\alpha}\right) \bar{\partial}_{\nu} \partial_{\mu}-\delta_{\mu \nu} \sum_{\rho} \bar{\partial}_{\rho} \partial_{\rho}\right] \delta_{x y}
$$

The matrix $L_{x y}^{\mu \nu}$ has a zero eigenvalue with $x$-independent eigenstates $\phi_{\mu}$. Correspondingly, the free propagator is ill-defined at zero momentum.

The origin of the problem is quite general : if the solutions of the classical equations of motion depend on some continuum parameter then the gaussian

\footnotetext{
${ }^{1} S_{F P}$ does not contribute in the gaussian approximation.
} 
action has zero modes [5, 6]. The perturbative scheme described above corresponds to the expansion about the constant solution $\theta_{x \mu}^{c l}=0$ of the classical equation of motion

$$
\frac{\partial S(\theta)}{\partial \theta_{x \mu}}=\frac{2}{g^{2}} \sum_{\nu} \bar{\partial}_{\nu} \sin \theta_{x ; \mu \nu}=0 .
$$

However, there is a continuum of solutions $\theta_{x \mu}^{c l}=\phi_{\mu}$ with different $\phi_{\mu}$ ?. The chosen gauge condition - Lorentz gauge - does not lift this degeneracy, and this produces the residual zero modes of the quadratic form $S_{\text {eff }}^{(0)}$.

The usual way to handle this problem is to exclude the $p=0$ mode from all Feynman diagramms (see, e.g., [7]), which is justified in the case of gaugeinvariant objects (e.g. Wilson loops) [3]. This is equivalent to the calculation of another average $\langle\Phi\rangle^{\prime}$ :

$$
\langle\Phi\rangle^{\prime}=\frac{1}{Z} \int\left[d \theta_{x \mu}\right] \delta\left(\sum_{x} \theta_{x \mu}\right) \cdot \Phi(\theta) \cdot e^{-S_{e f f}(\theta)} .
$$

To calculate $\langle\Phi\rangle$ perturbatively one should keep the zero-momentum mode under control in the perturbation expansion. It can be achieved by repeating the Faddeev-Popov trick

$$
1=\left.J_{0} \int\left[d \phi_{\mu}\right] \exp \left\{-\frac{1}{\epsilon} \sum_{\mu}\left(\phi_{\mu}-\frac{1}{V_{4}} \sum_{x} \theta_{x \mu}\right)^{2}\right\}\right|_{\epsilon \rightarrow 0} ; \quad\left[\phi_{\mu}\right]=\prod_{\mu=1}^{4} d \phi_{\mu},
$$

and making the change of variables $\theta_{x \mu}=\phi_{\mu}+g A_{x \mu}$. The average functional $\langle\Phi\rangle$ can be now represented in the form

$$
\langle\Phi\rangle \sim \int_{-\pi}^{\pi}\left[d \phi_{\mu}\right] \int\left[d A_{x \mu}\right] \prod_{\mu=1}^{4} \delta\left(\sum_{x} A_{x \mu}\right) \Phi(\phi+g A) e^{-S_{e f f}(\phi+g A)}=\int_{-\pi}^{\pi}\left[d \phi_{\mu}\right]\langle\Phi\rangle(\phi) .
$$

In eq.(14) one can safely expand in powers of $g^{2}$ and extend the limits of integration of $A_{x \mu}$ to $[-\infty ; \infty]$. The zero-momentum modes are not gaussian, and the integration over $\phi_{\mu}$ in eq.(14) should stay compact.

\footnotetext{
${ }^{2}$ There are also non-constant solutions of the eq.(11) which are not discussed in this paper (see [8]).
} 
The difference between the two kinds of averaging - $\langle\Phi\rangle^{\prime}$ and $\langle\Phi\rangle-$ manifests itself even in the lowest (gaussian) approximation. Let us calculate the photon correlator

$$
\Gamma_{\mu}(\tau)=\left\langle\mathcal{O}_{\mu}(\tau) \mathcal{O}_{\mu}(0)\right\rangle ; \quad \mathcal{O}_{\mu}(\tau)=\sum_{\vec{x}} \sin \theta_{x \mu} .
$$

Evidently, $\left\langle\mathcal{O}_{\mu}\right\rangle=0$. Ruling out the zero-momentum mode as in eq.(12) one obtains for $\tau=x_{4}$

$$
\begin{aligned}
\Gamma_{i}^{\prime}(\tau) & =\frac{V_{3}^{2}}{2 V_{4}} \sum_{p_{4} \neq 0} \frac{e^{i \tau p_{4}}}{4 \sin ^{2} \frac{p_{4}}{2}} ; \quad i=1 ; 2 ; 3 ; \\
\Gamma_{4}^{\prime}(\tau) & =0 \quad \text { at } \quad \alpha=0,
\end{aligned}
$$

where $V_{3}=N_{1} N_{2} N_{3}$. The most important observation concerning $\Gamma_{i}^{\prime}(\tau)$ is that it is not zero. It has a rather non-trivial dependence on $\tau$ and can even become negative.

Taking into account the zero-momentum modes $\phi_{\mu}$ one obtains in the Lorentz gauge

$$
\begin{aligned}
& \Gamma_{i}(\tau ; \phi)=C^{2}+\frac{V_{3}^{2}}{2 V_{4}} \cos ^{2} \phi_{i} \sum_{p_{4} \neq 0} \frac{e^{i \tau p_{4}}}{4 \sin ^{2} \frac{p_{4}}{2}} ; \\
& \Gamma_{4}(\tau ; \phi)=C^{2} ; \quad C^{2}=\frac{V_{3}^{2}}{g^{2}} \sin ^{2} \phi_{i}\left(1-b g^{2}\right)
\end{aligned}
$$

wheree $b \simeq 0.058$. The non-zero value of $\Gamma_{4}(\tau)$ is the indicator of the presence of zero-momentum modes.

The $\tau$-dependence of the correlator $\Gamma_{i}(\tau)$ can mimic the non-zero photon mass $\tilde{m}(\tau)$ if defined in accordance with eq.(3). As an example, in Figure 1 a one can see two correlators $\Gamma_{1}^{\prime}(z)$ and $\Gamma_{1}(z)$ with separation along the spacelike direction $z=x_{3}$ calculated on a $32^{3} \times 8$ lattice. The zero-momentum mode was chosen to be $\phi_{1}=0.01$ and $g^{2}=1$. The effective mass $\tilde{m}(z)$ corresponding to the correlator $\Gamma_{1}(z ; \phi)$ is shown in Figure 1]b. Of course, one can hardly expect the appearence of the nonzero magnetic mass in the Coulomb phase in the pure gauge $U(1)$ theory. It is rather a 'phantom' produced by a) the non-trivial $\tau$-dependence of the correlator $\Gamma^{\prime}(\tau)$ and b) the zero-momentum modes. 


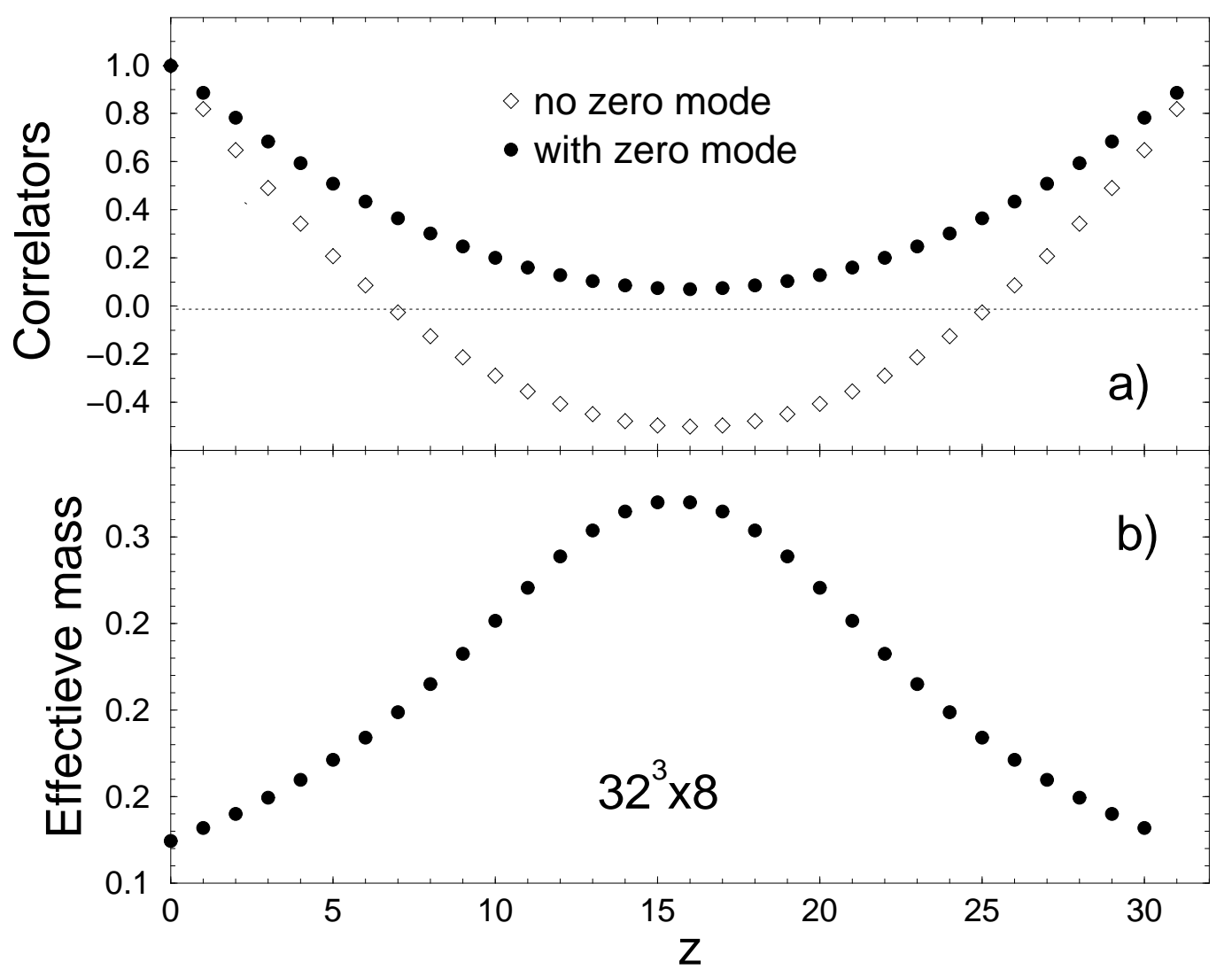

Figure 1: The correlators $\Gamma_{1}^{\prime}(z)$ and $\Gamma_{1}(z)(\mathbf{a})$ and the effective ('magnetic') mass $\tilde{m}(z)(\mathbf{b})$ on a $32^{3} \times 8$ lattice. The zero-momentum mode $\phi_{1}$ was chosen to be $\phi_{1}=0.01$, and $g^{2}=1$.

The extension to the case of the non-abelian theories is straightforward. As well as in the abelian case, there is a degeneracy of the $x$-independent solutions $U_{\mu}^{c l}$. of the classical equations of motion due to the toroidal structure of the periodic lattice [9, 3]. These solutions have zero action if $\left[U_{\mu}^{c l .}, U_{\nu}^{c l .}\right]=$ 0 , they are called torons. An example of a toron is $U_{\mu}^{c l .}=\exp \left\{i \phi_{\mu} T\right\}$, where $T$ is one of the generators of the gauge group and $\phi_{\mu}$ are four numbers. The perturbative expansion deals with fluctuations about the torons.

Suppose for simplicity that $U_{\mu}^{c l}=\exp \left\{i \phi_{\mu} T\right\}$ is a diagonal matrix. One can represent the link field in the form $U_{x \mu}=\exp \left\{i A_{x \mu}\right\} \exp \left\{i \phi_{\mu} T\right\}$ where $A_{x \mu}$ satisfy the condition $\sum_{x} A_{x \mu}^{\text {diag. }}=0$. The non-abelian field $A_{x \mu}=\vec{A}_{x \mu} \vec{T}$ 
has the non-diagonal zero-momentum modes $\bar{A}_{\mu}=A_{\mu}(p=0)$, and their contribution to the effective action in the lowest approximation is

$$
S_{e f f}^{(0 ; \text { zero modes })} \sim \sum_{\mu \nu} \operatorname{Tr}\left\{\left(D_{\mu} \bar{A}_{\nu}-D_{\nu} \bar{A}_{\mu}\right)^{2}\right\},
$$

where $D_{\mu} A_{\nu}=\exp \left\{i \phi_{\mu} T\right\} A_{\nu} \exp \left\{-i \phi_{\mu} T\right\}-A_{\nu}$. In principle, torons are not physically equivalent. If $U_{\mu}^{c l} \in Z_{N}$ (singular toron) then $D_{\mu} \bar{A}_{\nu}=0$, and the zero-momentum modes are not gaussian, as in the $U(1)$ case. Careful consideration shows that the zero-momentum corrections to the Wilson loops are rather small [3].

On the contrary, the dependence of the gauge--variant correlators $\Gamma_{\mu}(\tau)$ on the zero-momentum can be very strong. The chosen gauge-fixing does not prohibit the fluctuations about some constant value $\phi_{\mu}^{a}$, and the shift $A_{x \mu}^{a} \rightarrow A_{x \mu}^{a}+\phi_{\mu}^{a} \quad$ (for small fields) produces the appearence of the nonnegative constant term in the correlator as it happens in the $U(1)$ theory (compare eq.(16) and eq.(17)).

The main conclusion is that the contribution of the zero-momentum modes to the zero-momentum gauge-variant correlators cannot be ignored. Even rather small zero-momentum modes $\left(\left|\phi_{\mu}\right| \ll 1\right)$ can become very competitive when compared with the 'normal' modes, affecting strongly the effective masses.

It would be interesting to know how the zero-momentum modes 'develop' during the updating procedure, and what their dependence on the chosen gauge is. These questions deserve a furthur study.

From the practical point of view, presumably, it is preferable to generate configurations in $\mathrm{MC}$ calculations suppressing the zero-momentum modes as in eq. (12).

\section{Acknowledgements}

I would like to express my gratitude to U. Heller for useful discussions.

\section{References}


[1] J.E. Mandula and M. Ogilvie, Phys. Lett. 185B (1987) 127.

[2] U. Heller, F. Karsch and J. Rank, Phys. Lett. 355B (1995) 511.

[3] A. Coste, A. Gonzalez-Arroyo, J. Jurkiewicz and C. P. Korthals Altes, Nucl. Phys. B262 (1985) 67.

[4] I. Montvay and G. Münster, Quantum Fields on a Lattice, Cambridge University Press, 1994.

[5] A. M. Polyakov, Nucl. Phys. B120 (1977) 429.

[6] D. Amati and A. Rouet, Phys. Lett. B73 (1978) 39.

[7] U. Heller and F. Karsch, Nucl. Phys. B251 (1985) 254.

[8] V.K. Mitrjushkin, in preparation.

[9] A. Gonzalez-Arroyo, J. Jurkiewicz and C. P. Korthals Altes, Proc. 11th NATO Summer Institute, Freiburg, 1981, eds. J. Honerkamp et al. (Plenum, NY, 1982). 